\title{
Parallels among the "music scores" of solar cycles, space weather and Earth's climate
}

\author{
Zoltán Kolláth ${ }^{1}$, Katalin Oláh ${ }^{1}$ and Lidia van Driel-Gesztelyi ${ }^{1,2,3}$ \\ ${ }^{1}$ Konkoly Observatory, Budapest, Hungary \\ email: kollath@konkoly.hu \\ ${ }^{2}$ Observatoire de Paris, LESIA, CNRS, UPMC Univ. Paris 06, \\ Univ. Paris-Diderot, Meudon, France \\ ${ }^{3}$ University College London, Mullard Space Science Laboratory, UK
}

\begin{abstract}
Solar variability and its effects on the physical variability of our (space) environment produces complex signals. In the indicators of solar activity at least four independent cyclic components can be identified, all of them with temporal variations in their timescales.

Time-frequency distributions (see Kolláth \& Oláh 2009) are perfect tools to disclose the "music scores" in these complex time series. Special features in the time-frequency distributions, like frequency splitting, or modulations on different timescales provide clues, which can reveal similar trends among different indices like sunspot numbers, interplanetary magnetic field strength in the Earth's neighborhood and climate data.

On the pseudo-Wigner Distribution (PWD) the frequency splitting of all the three main components (the Gleissberg and Schwabe cycles, and an $\approx 5.5$ year signal originating from cycle asymmetry, i.e. the Waldmeier effect) can be identified as a "bubble" shaped structure after 1950. The same frequency splitting feature can also be found in the heliospheric magnetic field data and the microwave radio flux.
\end{abstract}

Keywords. methods: data analysis, Sun: activity, Sun: magnetic fields, solar-terrestrial relations

\section{Time-frequency representations}

Individual peaks in Fourier spectra have physical meaning only for very specific type of data (e.g. signals with constant periods). In other cases time-frequency distributions (TFD) should be investigated. The most frequently used time-frequency methods are the wavelet and the Short Time Fourier Transform (see e.g. Kolláth \& Oláh 2009), however more sophisticated representations exist based on the Wigner distribution (Wigner 1932; Cohen 1995). The generalised time-frequency distribution is given by

$$
\begin{aligned}
C(t, f)= & \frac{1}{2 \pi} \iiint \exp (-i \xi t-2 \pi i \tau f-i \xi \theta) \\
& \Phi(\xi, \tau) s^{*}(\theta-\tau / 2) s(\theta+\tau / 2) d \theta d \tau d \xi
\end{aligned}
$$

where $s(t)$ is the analysed time series and $\Phi(\xi, \tau)$ is the kernel of the distribution that determines the specific properties of the distribution. The Wigner-Ville transformation is given by $\Phi(\xi, \tau)=1$. This distribution is heavily contaminated by cross terms of the different components. A bi-Gaussian kernel defines the pseudo-Wigner distribution:

$$
\Phi(\xi, \tau)=\exp \left(-\xi^{2} / \beta^{2}-\tau^{2} / \alpha^{2}\right),
$$

where $\alpha$ and $\beta$ are parameters that contoll the properties of the distribution.

The figures of time-frequency distributions are colour/grey-scale coded: the darker the shade, the larger the amplitude. "Bubbles" in TFDs are signs of temporary frequency splittings indicating the appearance of additional periodicities. Figure 1 displays 
the Short-Term Fourier Transform (left) and the pseudo-Wigner Distribution (right) of a test signal with constant frequencies indicated by horizontal lines. The $0.09 \mathrm{c} / \mathrm{y}(11.1 \mathrm{y})$ component is always present, while an $\mathrm{f}=0.115 \mathrm{c} / \mathrm{y}(8.7 \mathrm{y})$ component appears at about 1950, which exists only for a limited time interval. This signal results in bubbles in most of the time-frequency representations. Frequency modulation or other non-stationarity effects may further modify these structures.

\section{The Waldmeier effect on time-frequency representations}

Parallels and differences of the different periodic components within the same dataset, like modulations of the harmonics of the frequency of the 11-year solar cycle, provide additional clues for understanding processes like the Waldmeier effect.

Waldmeier (1935) has shown that an anti-correlation exists between the rise times of sunspot cycles and their strengths (i.e. shorter rise-times are coupled with higher cycle maxima). Shorter rise-times imply faster rise-rates. Thus the Waldmeier effect can also be formulated as a correlation between rise-rates and cycle strengths. The Waldmeier effect is a robust feature of the solar cycle, with consequences on correlations of other cycle measures and even cycle forecast.

In our time-frequency diagram (Fig. 2) the Waldmeier effect appears as a higher than expected amplitude of the half period of the Schwabe cycle, showing clearly the strong asymmetry of the higher amplitude (stronger) cycles.

Petrovay (2009), using harmonic analysis, has shown that the inverse correlation between cycle length and amplitude is the consequence of the Waldmeier effect, i.e. the strong inverse correlation between rise time and cycle amplitude, since the decay times do not correlate with cycle strengths. Cameron \& Schüssler (2007) showed an important implication of the Waldmeier effect: since stronger cycles tend to rise faster to their maximum activity, the temporal overlapping of cycles leads to a shift of the minimum epochs that depends on the strength of the following cycle. This information is picked up by precursor methods, widely used for cycle-to-cycle forecasting. However, their finding indicates that the precursors are ultimately a simple consequence of the Waldmeier effect.

\section{Solar activity variations}

The presence of similar patterns at different frequencies, e.g. the bubbles at 5-, 10and 80-year cycle lengths during the last 60 years (Fig. 2), provide very strong indication of multiple cycles in solar activity. Data noise cannot possibly produce such coherent multi-frequency patterns. The long-term variation of solar activity is a combination of at least two different cycles with variable lengths and amplitudes. This complex frequencysplitting pattern seems to be an important potential precursor of extreme cycle phases like long, deep minima and grand maxima of solar activity.
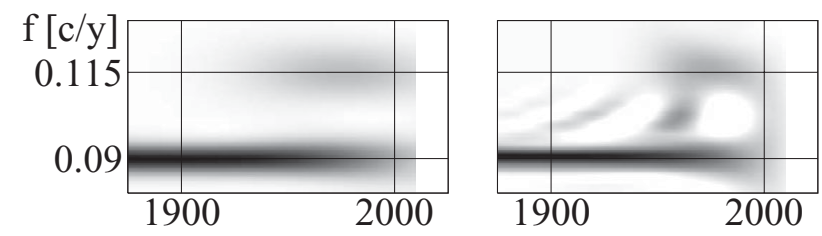

Figure 1. Short-Term Fourier Transform (left) and the pseudo-Wigner Distribution (right) of the test signal. The frequencies are measured in cycles/year (c/y). 
Analyzing long-term data with such "music scores" can bring to light recurrent structures hidden in other data representations. These recurrent "tunes", due to their regular nature, can be used for forecasting the phenomena.

Solar activity went through several frequency shifts during the centuries covered by sunspot observations, like the one around 1700 (Fig. 2, left panel). This frequency shift event was strongly related to the termination of the Maunder minimum.

A similar structure can be found just before the Maunder minimum. The period splitting of the Gleissberg cycle in the 16th century, i.e., the appearance of an about 30 year long cycle together with the century-long variation looks the same as the recent period split, which started in 1950 . We recall that 400 years ago a similar feature was followed by the Little Ice Age.

Then it is straightforward to compare the "scores" of solar activity to climate data. For this comparison we selected a global dataset, the Monthly Global Ocean Temperature Anomalies (Smith et al. (2008), http://www.ncdc.noaa.gov/cmb-faq/anomalies.php) and local temperature observations consistently observed from Armagh (Butler et al. 2005). The Pseudo-Wigner distributions of the three data sets are displayed on Fig. 3. Here we used a higher maximum frequency, so even the third harmonic of the Schwabe cycle is visible (fourth bubble on the left diagram). There is no direct (frequency-to-frequency) agreement among the time-frequency distributions of the different data sets - even the different climate proxies show different structures. However, it should be noted that the appearance of the new frequencies in solar activity slightly before 1950 correlates with the changes of the frequency patterns in the global sea temperature data, i.e., a periodicity around 10 years appears with a deceasing trend (Fig. 3, lower middle panel). The result for the Armagh mean temperature (Fig. 3, lower right panel) has a hint of a bubble-structure starting at about the same time as found from the sunspot data.
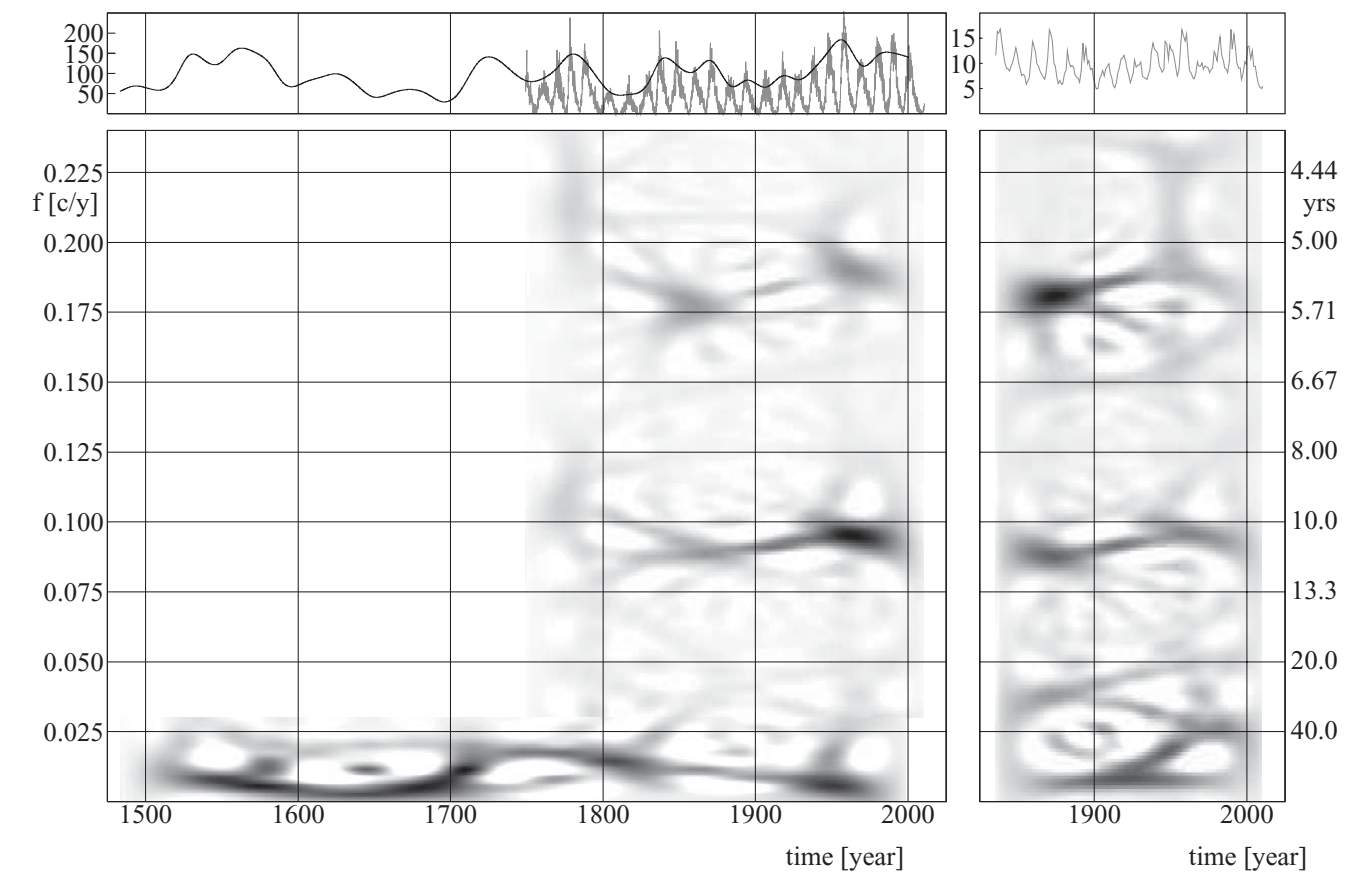

Figure 2. Left panels: Solar activity sunspot number (after 1750). Envelope: Schove (1955) data. Right panels: Heliospheric Magnetic Field - Svalgaard \& Cliver (2010). Lower panels: pseudo-Wigner ditributions of the datasets. 

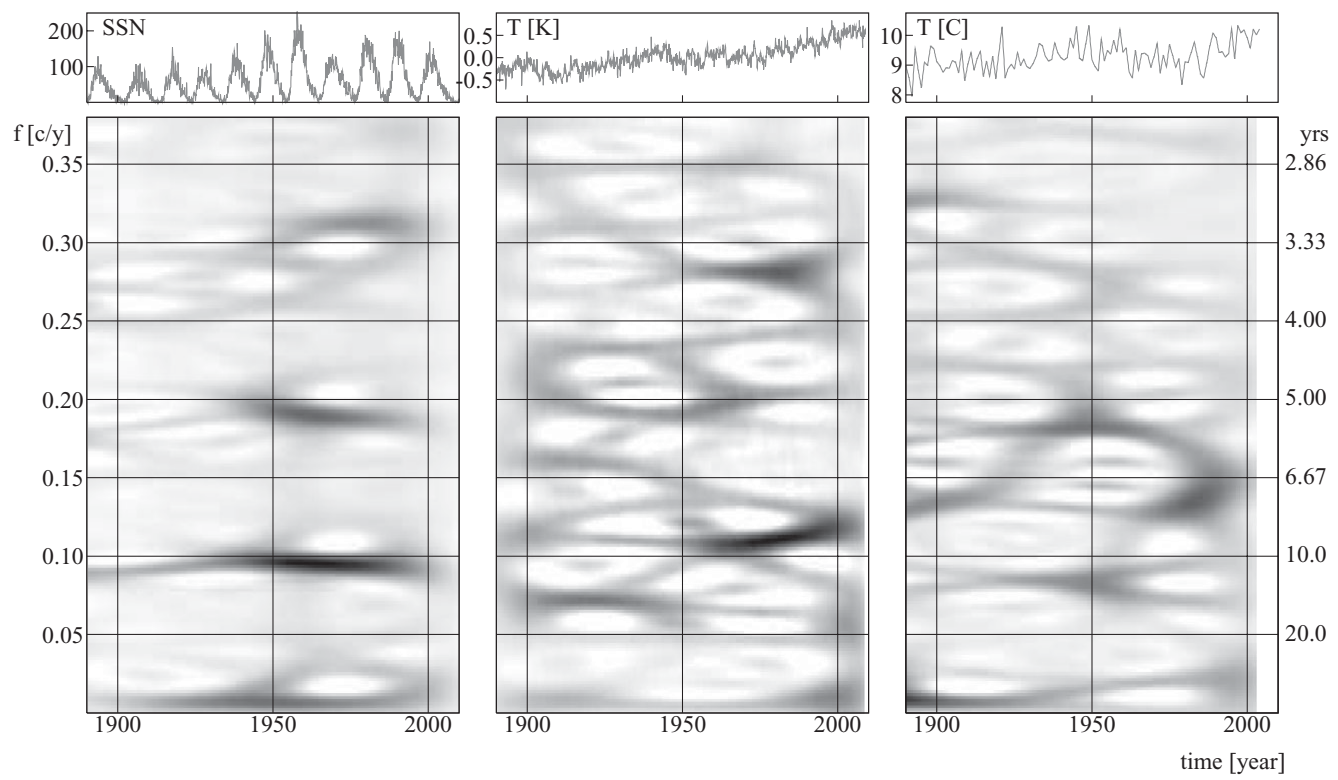

Figure 3. Left panel: Solar activity (sunspot number). Middle panel: The monthly global ocean temperature anomalies. Right panel: Armagh mean temperatures. Upper panels: Datasets. Lower panels: Pseudo-Wigner distributions of the datasets.

\section{Acknowledgements}

This work was supported by the Hungarian OTKA grant K-81421.

\section{References}

Butler, C. J., García-Suárez, A. M., Coughlin, A. D., \& Morrel, C. 2005, Int. J. Climatol., 25, 1055 (see also: Armagh Observatory Climate Series Vol. 2)

Cameron, R. \& Schüssler, M. 2007, ApJ, 659, 801

Cohen, L. 1995, Time-Frequency Analysis, (Prentice-Hall, Englewood Cliffs).

Kolláth, Z. \& Oláh, K. 2009, A\&广A, 501, 695

Petrovay, K. 2009, in A. G. Kosovichev, A. H. Andrei \& J.-P. Rozelot (Eds) Solar and Stellar Variability: Impact on Earth and Planets Proc IAU Symp., No. 264, p. 150.

Schove, D. J. 1955, J. Geophys Res., 60, 127

Smith, T. M., Reynolds, R. W., Peterson, T. C., \& Lawrimore, J. 2008, J. Climate, 21, 2283

Svalgaard, L. \& Cliver, E. W. 2010, J. Geophys. Res., 115, A09111

Waldmeier M. 1935, Mitt. Eidgen. Sternw. Zurich, 14, 105

Wigner, J. 1932, Phys. Rev., 40, 749 